\title{
Complete Radiological and Metabolic Response In MCRC
}

\author{
Asmaa Elhussainy*, Asit Mohanty, Jaroslav Nemec and Jasem Al barrak \\ Medical oncology department, Kuwait Cancer Control Center, Kuwait
}

Submission: February 06, 2020; Published: February 13, 2020

*Correspondence Author: Asmaa Elhussainy, Medical oncology department, Kuwait Cancer Control Center, Kuwait

\begin{abstract}
Background: Complete Response in metastatic colon cancer after chemotherapy alone is uncommon, even patients with radiological CR after chemotherapy harbor persistent macroscopic or microscopic residual disease in more than $80 \%$ of the cases 5 years survival in Stage VI colon cancer is less than $10 \%$. We are going to present a case with mCRC that achieved CR and finished 5 years of follow up.

Case presentation: A 54-year-old female patient, known to be hypertensive was diagnosed as a case of metastatic colon cancer to liver, peritoneum with omental cakes and abdominal lymph nodes involvement. Received chemotherapy that achieved complete radiological response and finished her follow up for 5 years with no evidence of disease recurrence.

Conclusion: CR to systemic chemotherapy is uncommon, but this case demonstrates that it can be durable in patients with colorectal cancer with extensive metastasis. This case report can indicate that some patients with mCRC can be cured with systemic therapy only challenging the prevailing paradigm of mCRC therapy.

Keywords: Colon cancer; Metastatic; Complete response; Chemotherapy
\end{abstract}

\section{Introduction}

Colorectal cancer (CRC) is one of the most common cancer diagnoses worldwide, and approximately $20 \%$ of new diagnoses are metastatic at presentation [1]. The majority of cases of metastatic colorectal cancer (mCRC) are incurable. Systemic chemotherapy is the treatment of choice in patients with unresectable metastatic colon cancer. The current management of disseminated metastatic colon cancer involves various active drugs, either in combination or as single agents. And although it is often used with palliative intent, it may be used in an attempt to achieve a complete response [2]. The choice of therapy is based consideration of the goals of therapy, the type and timing of prior therapy, the mutational profile of the tumor, and the differing toxicity profiles of constituent drugs. Approximately $17 \%$ of patients with metastatic colorectal cancer have peritoneal spread, with $2 \%$ having the peritoneum as the only site of metastasis. Peritoneal dissemination is one of the leading causes of death associated with colon cancer recurrence and is often challenging to treat [3]. patients with peritoneal metastasis generally have a shorter PFS and OS than those without peritoneal involvement [4]. The goal of treatment for most abdominal and peritoneal metastasis is palliative rather than curative and primary consist of systemic therapy with palliative surgery or stenting if needed for obstruction or impending obstruction [5] the median survival with optimal therapy is now almost 3 years. Despite improvements in systemic therapy for mCRC, long -term survival and complete radiologic Reponses remain rare [6]. During this case we are emphasizing a marvels response to chemotherapy in extensive disease spread.

\section{Case Presentation}

Our patient is a 54 ys old female, known to be hypertensive on medical treatment. August 2012 was investigated for uncontrolled abdominal pain, anemia and asthenia, where CT scan abdomen and pelvis showed a mass at caecum and ascending colon with multiple peritoneal nodules, omental cakes with two hepatic focal lesions (largest $15 \mathrm{~mm})$ and large exo phytic splenic lesion $(9.5 \mathrm{x}$ $7.5 \mathrm{~cm}$ ) (Figure 1). Her blood investigation showed $\mathrm{HB}=8.3 \mathrm{gm} / \mathrm{dl}$, tumor markers $(\mathrm{CEA}=220)$ Colonoscopy biopsy from cecal mass showed adenocarcinoma. She was in good condition PS =0 ECOG, we embarked her on CAPOX protocol (oxaliplatin 130/m2 D1 + 


\section{Cancer Therapy \& Oncology International Journal}

capecitabine $1000 \mathrm{mg} / \mathrm{m} 2$ twice daily for 14 days, to be repeated every 3 ws) along with Cetuximab from 3rd cycle as KRAS status was wild type. December 2012: CT scan post 3 months treatment showed regressive course of her disease regarding all above involved organs, tumor marker (CEA= 7.19) (Figure 2).

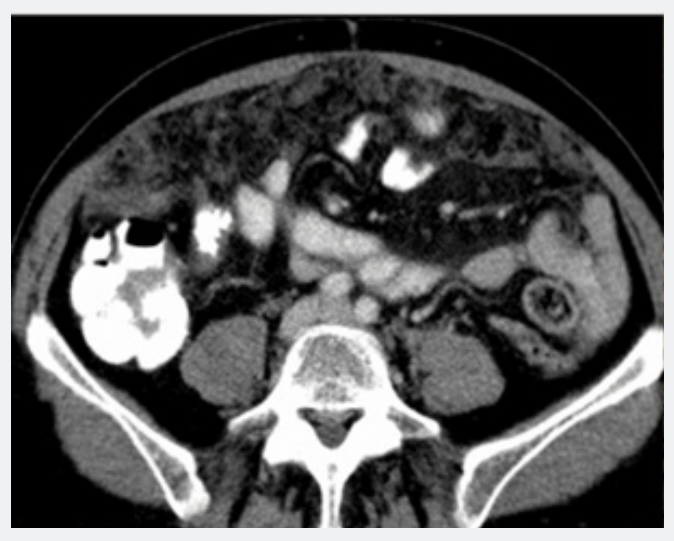

Figure 1: CT scan abdomen and pelvis showing a mass at caecum and ascending colon with multiple peritoneal nodules, omental cakes with two hepatic focal lesions (largest $15 \mathrm{~mm}$ ) and large exo phytic splenic lesion $(9.5 \times 7.5 \mathrm{~cm})$
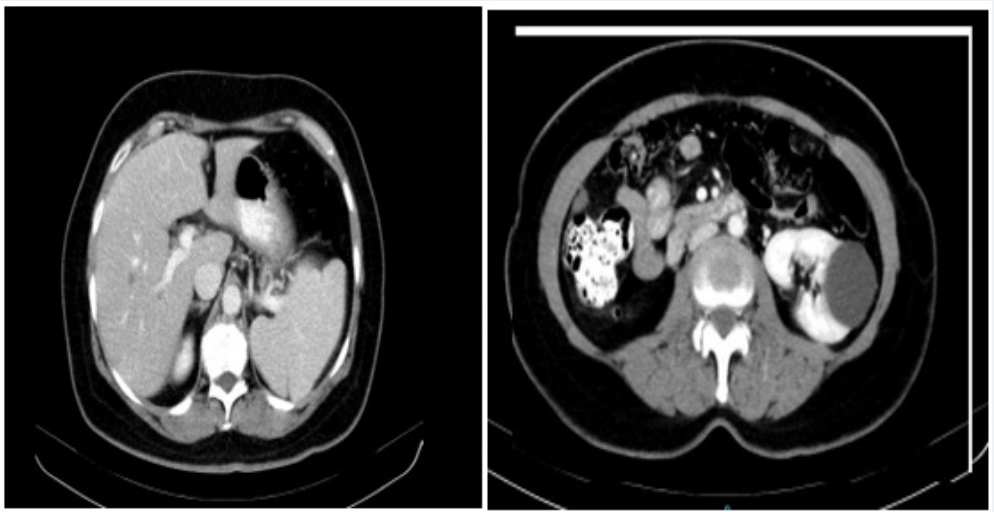

Figure 2: CT scan post 3 months treatment showing regressive course of her disease regarding all above involved organs, tumor marker (CEA= 7.19).

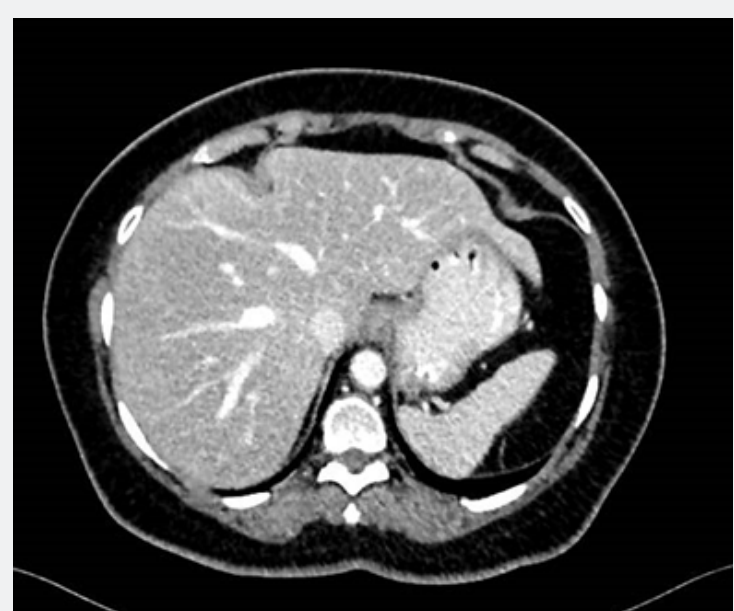

Figure 3: PET/ CT scan showing complete metabolic response apart from FDG uptake at rectum SUV 7.5 


\section{Cancer Therapy \& Oncology International Journal}

She received 10 courses of that protocol with G I skin rash and G II oral mucositis that was under good control by local measures with doxycycline. July 2013: CT scan showed no evidence of her disease regarding her colonic thickening, hepatic focal lesions, peritoneal deposits, omental cakes, abdominal or mesenteric lymph nodes, while her splenic lesion remained unchanged. Tumor marker (CEA = 0.7). At that time, we discussed with patient's daughter to modify her mother's treatment for maintained oral capecitabine only and preserve oxaliplatin till time of disease recurrence and she agreed for this. September 2013: PET/ CT scan showed complete metabolic response apart from FDG uptake at rectum SUV 7.5 (Figure 3). She refused to proceed for colonoscopy, so MRI pelvis was done with no evidence of abnormal masses or disease recurrence (Figure 4). November 2013: after 7 courses of capecitabine+ cetuximab CT scan again showed no evidence of disease recurrence or metastasis. May 2014 after 14 cycle of capecitabine+ cetuximab, CT scan again for the 3rd time documenting no evidence of disease recurrence or metastasis. Tumor marker was normal (CEA=0.6). At that time the decision was to keep her under wait and watch. She continued under regular follow up with wait and watch policy till August 2018 where she completed 5 years of regular follow up with no evidence of disease recurrence by CT scan and normal tumor marker.

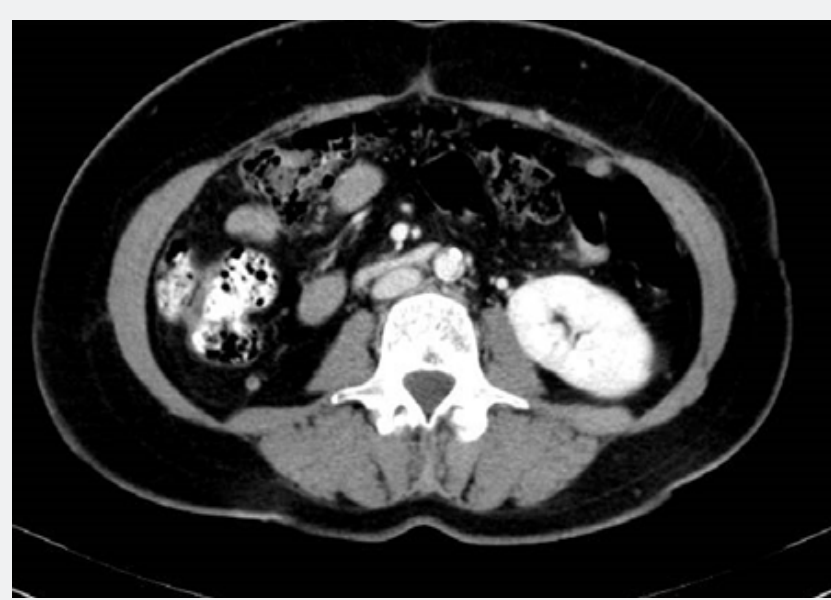

Figure 4: MRI pelvis with no evidence of abnormal masses or disease recurrence.

\section{Discussion}

Metastatic CRC usually represents an incurable situation, for which systemic chemotherapy in combination with targeted therapy is the treatment of choice [7,8]. Recent studies have shown that there is a role for EGFR inhibitors such as cetuximab and panitumumab along with chemotherapy in the first-line setting [9-11]. The CRYSTAL trial revealed a progression-free survival (PFS) and overall survival (OS) benefit in patients treated with FOLFIRI plus cetuximab compared to FOLFIRI alone, a benefit which was even greater when assessed in patients with RAS WT tumors $[12,13]$. The median survival with an EFGR inhibitor plus chemotherapy approaches 3 years; however, complete radiologic responses and long-term survival are rare.

In the FIRE-3 trial, patients with mCRC who received cetuximab with chemotherapy had an improved OS compared to those who received bevacizumab with chemotherapy [14]. It should be noted that this study did not meet its primary endpoint of improvement in overall response rate. The CALGB/ SWOG 80405 trial has since attempted to add clarity to the question of the optimal targeted therapy in the first-line setting. There was no difference in OS or PFS whether patients received cetuximab or bevacizumab in addition to first-line chemotherapy. A retrospective analysis, however, showed that patients with leftsided primary tumors had a better OS compared to those with right-sided tumors [15]. In those with left-sided tumors, OS was better for those who received cetuximab in combination with chemotherapy, whereas those with right-sided primary tumors had a better OS with bevacizumab and chemotherapy compared to cetuximab and chemotherapy.

Despite improved OS with the use of EGFR inhibitors in combination with chemotherapy, complete responses to systemic therapy remain rare. In the PEAK trial, $2 \%$ of patients had a complete radiologic response [16]. In the second-line setting, FOLFIRI and panitumumab resulted in a complete response in only one patient [17]. Further details regarding durability of response and sidedness of the primary tumor in these studies are not known; however, achieving a complete response is nonetheless a rare occurrence in patients with mCRC treated with palliative intent chemotherapy and Anti-EGFR. The prognostic and predictive significance of tumor sidedness is proved already since 2016 after the CALGB/SWOG 80405 trial But Our patient had a right sided RAS WT colon cancer and experienced an excellent 


\section{Cancer Therapy \& Oncology International Journal}

response with first-line chemotherapy plus an EGFR inhibitor. She received her treatment started in 2012 so at that time we gave her EGFR inhibitors based on RAS status .

A literature review confirms that durable complete responses in patients with $\mathrm{mCRC}$ are rare. Two case reports of long-term disease-free survival were identified from Japan. Although our patient had metastatic disease identified from beginning as extensive peritoneal and omental disease with liver lesions and therefore likely has a very high risk of recurrence, she has now survived over 7 years from the time of her initial diagnosis and currently has no radiologic evidence of disease. Nonetheless, she has had an excellent response to first-line therapy and has many further systemic therapy options available if her disease recurs in the future. As far as we are aware, there are no published case reports of durable complete responses with the use of a first-line EGFR inhibitor and chemotherapy in mCRC.

\section{Conclusion}

CR to systemic treatment is uncommon, but this case report demonstrates that it can be durable in patients with colorectal cancer with liver and peritoneal metastases. This case report indicates that some patients with $\mathrm{mCRC}$ can be cured with systemic therapy only, challenging the prevailing paradigm of mCRC therapy. As contrary to current practice of effective therapy in right and left sided metastatic colon cancer the tumor biology is the also most important factor for response to treatment.

\section{References}

1. Adam R, Delvart, V, Pascal G, Valeanu A, Castaing D, et al. (2004) Resue surgery for unresectable colorectal liver metastases dowen staged by chemotherapy : a model to predict long term susvival. Ann surg 240(4): 644-657.

2. Franco J, Shi Q Goldman Cd, , Pockaj BA, Nelson GD, et al. (2012) Treatment of colorectal peritoneal carcinomatosis with systemic chemotherapy: a pooled analysis of north central cancer treatment group phase III trials N9741 and N9841. J Clin Oncol 30(3): 263-267.

3. Franco J, Shi Q Meyers JP, Maughan TS, Adams RA, et al. (2016) Prognosis of patients with peritoneal metastatic colorectal cancer given systemic therapy: an analysis of individual patient's data from prospective randomized trials from the Analysis and Research in Cancers of the Digestive System (ARCAD) database. Lancet Oncol 17(12): 1709- 1719.

4. Kaver YL, Leenders BJ, Creemers GJ, Rutten HJ, Verwaal VJ, et al. (2013) Addition of biological therapies to palliative to palliative chemotherapy prolonges survivsl in patients with peritoneal carcinomatosis of colorectal origin. Am J Clin Oncol 36(2): 157-161.

5. Van Hooft JE, van halsema EE, vanbiervliet G, Beets-Tan RG, DeWitt JM, et al. (2014) Self expanding metallic stents for obstructing colonic and extracolonic cancer: European society of gastrointestinal endoscopy (ESGE) Clinical Guidline. Gastrointes Endosc 80: 747-761 e 741- 775.
6. Stintzing S, Modest DP, Rossius L, Lerch MM, von Weikersthal LF, et al. (2016) FOLFIRI plus cetuximab versus FOLFIRI plus bevacizumab for metastatic colorectal cancer (FIRE3): a post-hoc analysis of tumor dynamics in the final RAS wild type subgroup of this randomized open label phase 3 trail. Lancet Oncol 17(10): 1426-1434.

7. Papamichael D, Audisio RA, Glimelius B, de Gramont A, Glynne-Jones $\mathrm{R}$, et al. (2015) Treatment of colorectal cancer in older patients: International Society of Geriatric Oncology (SIOG) consensus recommendations 2013. Ann Oncol 26( 3): 463- 476.

8. Edwards MS, Chadda SD, Zhao Z, Barber BL, Sykes DP (2012) A systematic review of treatment guidelines for metastatic colorectal cancer. Colorectal Dis 14( 2): 31- 47.

9. Schwartzberg LS, Rivera F, Karthaus M, Fasola G, Canon JL, et al. (2014) PEAK: a randomized, multicenter phase II study of panitumumab plus modified fluorouracil, leucovorin, and oxaliplatin (mFOLFOX6) or bevacizumab plus mFOLFOX6 in patients with previously untreated, unresectable, wild-type KRAS exon 2 metastatic colorectal. J Clin Oncol 32(21): 2240- 2247.

10. Elez E, Argilés G, Tabernero J (2015) First-line treatment of metastatic colorectal cancer: interpreting FIRE-3, PEAK, and CALGB/SWOG 80405. Curr Treat Options Oncol 16(11): 52.

11. Douillard JY, Siena S, Cassidy J, Tabernero J, Burkes R, et al. (2014) Final results from PRIME: randomized phase III study of panitumumab with FOLFOX4 for first-line treatment of metastatic colorectal cancer. Ann Oncol 25(7): 1346- 1355.

12. Van Cutsem E, Köhne CH, Hitre E, Zaluski J, Chang Chien CR, et al. (2009) Cetuximab and chemotherapy as initial treatment for metastatic colorectal cancer. N Engl J Med 360(14): 1408-1417.

13. Van Cutsem E, Köhne CH, Láng I, Gunnar F, Marek P Nowacki, et al. (2011) Cetuximab plus irinotecan, fluorouracil, and leucovorin as first-line treatment for metastatic colorectal cancer: Updated analysis of overall survival according to tumor KRAS and BRAF mutation status. J Clin Oncol 29(15): 2011- 2019.

14. Stintzing S, Modest DP, Rossius L, Lerch MM, Von Weikersthal LF, et al. (2016) FOLFIRI plus cetuximab versus FOLFIRI plus bevacizumab for metastatic colorectal cancer (FIRE-3): a post-hoc analysis of tumour dynamics in the final RAS wild-type subgroup of this randomised open-label phase 3 trial. Lancet Oncol 17(10): 1426- 1434.

15. Venook AP, Niedzwiecki D, Innocenti F, et al. Impact of primary $\left(1^{\circ}\right)$ tumor location on overall survival (OS) and progression-free survival (PFS) in patients (pts) with metastatic colorectal cancer (mCRC): Analysis of CALGB/SWOG 80405 (Alliance). J Clin Oncol 34( 15 suppl): 3504.

16. Schwartzberg LS, Rivera F, Karthaus M, Fasola G, Canon JL, et al. (2014) PEAK: a randomized, multicenter phase II study of panitumumab plus modified fluorouracil, leucovorin, and oxaliplatin (mFOLFOX6) or bevacizumab plus mFOLFOX6 in patients with previously untreated, unresectable, wild-type KRAS exon 2 metastatic colorectal. J Clin Oncol 32( 21): 2240- 2247.

17. Peeters M, Price TJ, Cervantes A, Sobrero AF, Ducreux M, et al. (2010) Randomized phase III study of panitumumab with fluorouracil leucovorin, and irinotecan (FOLFIRI) compared with FOLFIRI alone as second-line treatment in patients with metastatic colorectal cancer. J Clin Oncol 28(31): 4706- 4713. 
This work is licensed under Creative Commons Attribution 4.0 License DOI: 10.19080/CTOIJ.2020.15.555918

\section{Your next submission with Juniper Publishers} will reach you the below assets

- Quality Editorial service

- Swift Peer Review

- Reprints availability

- E-prints Service

- Manuscript Podcast for convenient understanding

- Global attainment for your research

- Manuscript accessibility in different formats ( Pdf, E-pub, Full Text, Audio)

- Unceasing customer service

Track the below URL for one-step submission https://juniperpublishers.com/online-submission.php 